Cite this: Phys. Chem. Chem. Phys., 2011, 13, 13238-13246

\title{
Phenomenological model of spin crossover in molecular crystals as derived from atom-atom potentials
}

\author{
Anton V. Sinitskiy, ${ }^{* a c}$ Andrei L. Tchougréeff ${ }^{* b c}$ and Richard Dronskowski ${ }^{b}$ \\ Received 20th February 2011, Accepted 25th May 2011 \\ DOI: $10.1039 / \mathrm{c1cp20440h}$
}

The method of atom-atom potentials, previously applied to the analysis of pure molecular crystals formed by either low-spin (LS) or high-spin (HS) forms (spin isomers) of Fe(II) coordination compounds (Sinitskiy et al., Phys. Chem. Chem. Phys., 2009, 11, 10983), is used to estimate the lattice enthalpies of mixed crystals containing different fractions of the spin isomers. The crystals under study were formed by LS and HS isomers of $\mathrm{Fe}(\mathrm{phen})_{2}(\mathrm{NCS})_{2}$ (phen = 1,10-phenanthroline), $\mathrm{Fe}(\mathrm{btz})_{2}(\mathrm{NCS})_{2}$ (btz $=5,5^{\prime}, 6,6^{\prime}$-tetrahydro- $4 H, 4^{\prime} H$-2,2' -bi-1,3-thiazine), and $\mathrm{Fe}(\mathrm{bpz})_{2}$ (bipy) $\left(\mathrm{bpz}=\operatorname{dihydrobis}\left(1\right.\right.$-pyrazolil)borate, and bipy $=2,2^{\prime}$-bipyridine). For the first time the phenomenological parameters $\Gamma$ pertinent to the Slichter-Drickamer model (SDM) of several materials were independently derived from the microscopic model of the crystals with use of atom-atom potentials of intermolecular interaction. The accuracy of the SDM was checked against the numerical data on the enthalpies of mixed crystals. Fair semiquantitative agreement with the experimental dependence of the HS fraction on temperature was achieved with use of these values. Prediction of trends in $\Gamma$ values as a function of chemical composition and geometry of the crystals is possible with the proposed approach, which opens a way to rational design of spin crossover materials with desired properties.

\section{Introduction}

Molecular crystals of coordination compounds of $d$-elements, capable of spin crossover transitions (SCT), are at present under active investigation. ${ }^{1-20}$ The average crystal field splitting parameter $10 D q$ in these crystals is comparable with the average pairing energy of the $d$-electrons which makes it possible that the high-spin (HS) and low spin (LS) forms have close energies and may coexist in a crystal. The external thermodynamical parameters, such as temperature or pressure, affect the equilibrium fractions of the spin forms. The process when the fraction of molecules of different total spin changes in response to changes of external conditions is precisely the SCT.

The materials exhibiting SCT are rather well studied experimentally, but the theoretical description of this phenomenon strongly lags behind. The simplest and most widespread model of SCT in solids was proposed by Slichter and Drickamer ${ }^{21}$ (the Slichter-Drickamer model, SDM). This model treats the solid undergoing a spin transition as a regular solution

${ }^{a}$ Department of Chemistry, The University of Chicago, Chicago, Illinois 60637,USA.E-mail: sinitskiy@uchicago.edu

${ }^{b}$ Institut für Anorganische Chemie, RWTH Aachen University, Landoltweg 1, 52056, Aachen, Germany.

E-mail: andrei.tchougreeff@ac.rwth-aachen.de

${ }^{c}$ Poncelet Laboratory, Independent University of Moscow,

Bolshoy Vlasyevskiy Pereulok 11, 119002, Moscow, Russia (mixture) of molecules in the LS and HS states. The Gibbs energy of the system is:

$$
G(x, T)=G(0, T)+x(\Delta H-T \Delta S)+\Gamma x(1-x)-T S_{\operatorname{mix}},
$$

where $x$ is the fraction of the HS molecules; $\Delta H$ and $\Delta S$ are the variations of the enthalpy and the entropy in the course of the SCT (they may be independently obtained from calorimetric measurements, like in ref. 22 , or phenomenologically fitted to describe the experimental $x(T)$ curve, like in ref. 23$) ; \Gamma$ is a phenomenological (effective) intermolecular interaction parameter, showing whether the molecules in the mixed crystal prefer to be surrounded by the molecules of the same $(\Gamma>0)$ or different $(\Gamma<0)$ spin; $S_{\mathrm{mix}}$ is the entropy of mixing:

$$
S_{\mathrm{mix}}=-R[x \ln x+(1-x) \ln (1-x)] .
$$

As usual, the equilibrium state at a given temperature $T$ is determined by the minimum of the Gibbs energy $G(x, T)$. Note that since the term $G(0, T)$ in eqn (1) is independent of $x$, it drops out from the formulas defining the equilibrium values of $x(T)$.

This model predicts, in agreement with the experiments, that the SCT may be either smooth or abrupt or may exhibit hysteresis, and this character is determined by the value of $\Gamma$, characteristic for each material. More specifically, SCT with $\Gamma>0$ are classified as cooperative and the corresponding $x(T)$ curves have a steeper slope in the transition region than one 
without interaction $(\Gamma=0)$. Those with $\Gamma<0$ are classified as anticooperative and the corresponding $x(T)$ curves have a gentler slope in the transition region than those without interaction. Among the cooperative transitions those with $\Gamma>\Gamma_{\mathrm{c}}=2 k_{\mathrm{B}} T_{\mathrm{c}}$ (where $T_{\mathrm{c}}=\Delta H / \Delta S$ ) refer to the first-order phase transition and thus are abrupt and may exhibit hysteresis. The key parameter determining qualitative features of SCT is thus $\Gamma$. It is an effective parameter arising from some averaging of energies of intermolecular interactions - in fact, over a nonspecifically wide range of these interactions. It was demonstrated in ref. 2 with a simple model intermolecular potential that such an averaging is nontrivial and that $\Gamma$ can be of either sign depending on details of the lattice relaxation.

A significant theoretical disadvantage of this and other existing models is that they contain phenomenological parameters, like $\Gamma$ in the SDM, that have not been calculated independently. The existing microscopic models use oversimplified description of the molecules (as spheres, ellipsoids, etc.), which prevent construction of a complete theory, especially due to importance of the short intermolecular contacts $(\pi-\pi$ interactions, S $\cdots \mathrm{H}-\mathrm{C}$ interactions, hydrogen bonds, etc. $\left.{ }^{1,3}\right)$ tentatively responsible for the cooperativity effects (see, however, section IV).

In this paper we try to use the explicit intermolecular potentials to estimate the adequacy of the Slichter-Drickamer model of SCT and to predict its phenomenological parameters. The method used in our previous work ${ }^{1}$ employs explicit potentials for intermolecular interactions - namely, the atomatom potentials. The underlying fact is that the crystals exhibiting SCT studied in the present work are formed by neutral molecules of coordination compounds with bulky organic ligands. Intermolecular contacts in such crystals are those between the organogenic atoms like $\mathrm{C}, \mathrm{H}, \mathrm{N}, \mathrm{S}$, etc. In this context, the atom-atom potentials, being the standard method developed for organic molecular crystals, can be successfully applied and yield good results in modeling the pure crystals formed by the individual LS and HS isomers.

The method of atom-atom potentials ${ }^{24}$ assumes that the energy of the molecular crystal per molecule (calculated relative to the energy of an isolated molecule) can be represented as:

$$
u=\frac{1}{2 Z} \sum_{\alpha \alpha^{\prime} m m^{\prime} \mathbf{r}} E_{\alpha \alpha^{\prime}}\left(R\left(\alpha \alpha^{\prime} m m^{\prime} \mathbf{r} \mathbf{0}\right)\right)
$$

where each term is the energy of the interaction between the $\alpha$-th atom of the $m$-th molecule in the unit cell number $\mathbf{r}=\left(r_{a}, r_{b}, r_{c}\right)$ and the $\alpha^{\prime}$-th atom of the $m^{\prime}$-th molecule in the unit cell $\mathbf{0}$ depending on the interatomic distance $R$; $Z$ is the number of molecules per unit cell. The most widespread approximations for the atom-atom interaction are the Buckingham potential:

$$
E_{\alpha \alpha^{\prime}}(R)=-\frac{A_{\alpha \alpha^{\prime}}}{R^{6}}+B_{\alpha \alpha^{\prime}} e^{-C_{\alpha \alpha^{\prime}} R}
$$

and the Lennard-Jones potential:

$$
E_{\alpha \alpha^{\prime}}(R)=-\frac{A_{\alpha \alpha^{\prime}}}{R^{6}}+\frac{B_{\alpha \alpha^{\prime}}}{R^{12}}
$$

where $A_{\alpha \alpha^{\prime}}, B_{\alpha \alpha^{\prime}}, C_{\alpha \alpha^{\prime}}$ are some method specific constants characterizing interactions of atoms $\alpha, \alpha^{\prime}$ as dependent on the chemical nature of the latter.

Through the dependence of $R\left(\alpha \alpha^{\prime} m m^{\prime} \mathbf{r} \mathbf{0}\right)$ on its arguments, the energy is a fast computable function of the lattice parameters $a, b, c, \alpha, \beta$ and $\gamma$, of relative positions and orientations of the molecules in the unit cell, provided the structure of each molecule is fixed. Having found the minimum of this function, one gets estimates of the intermolecular interaction energy (sublimation energy of the crystal), the equilibrium unit cell parameters, and the positions and orientation of the molecules in the unit cell.

Based on these theoretical concepts, we endeavor to construct phenomenological models of SCT in the molecular crystals. The rest of the article is designed as follows. In section II, we describe the input data used for modelling: the crystal structure of the compounds under study; the parameterization of the atom-atom potentials; the software used for the energy calculations; the experimental data on magnetic susceptibility providing information on the thermal dependence of the fractions of the spin isomers in the crystals. Section III includes description and discussion of our results concerning the parameters of SDM. Section IV provides the conclusion and discussion of relation with other microscopical models. Finally, the Appendix contains the comparative analysis of the enthalpies predicted by different versions of atom-atom potentials.

\section{Data collection}

The materials under study are formed by the spin isomers of $\mathrm{Fe}(\mathrm{phen})_{2}(\mathrm{NCS})_{2}, \mathrm{Fe}(\mathrm{btz})_{2}(\mathrm{NCS})_{2}$, or $\mathrm{Fe}(\mathrm{bpz})_{2}$ (bipy) molecules (for structures of the ligands see Fig. 1). All three materials consist of neutral molecules, and are held together by the van der Waals forces.

The MOLCRYST program suite ${ }^{25}$ capable of calculation and minimization of molecular crystals energy and enthalpy was employed. Three atom-atom potentials were used:

(1) the Buckingham potential with the set of parameters from ref. 26 (further referred to as 6-exp);

(2) the Lennard-Jones potential with the parameters taken from ref. 27 (further referred to as 6-12);

(3) the Buckingham potential with the parameters set derived in ref. 28; based on the set of parameters from
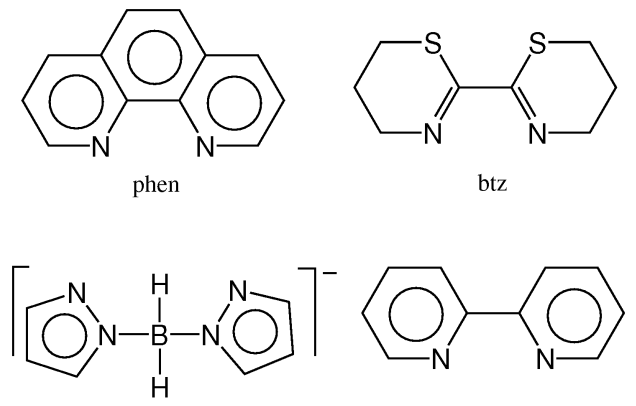

bpz

bipy

Fig. 1 Structure formulas for the ligands of the spin-active complexes studied. 
ref. 26 , but adjusted in ref. 1 to achieve a better reproduction of the equilibrium lattice parameters of the pure LS and HS crystals of the $\mathrm{Fe}(\text { phen })_{2}(\mathrm{NCS})_{2}$ and $\mathrm{Fe}(\mathrm{btz})_{2}(\mathrm{NCS})_{2}$ compounds and their variation throughout the SCT (further referred to as 6-expM).

The molecular structures of the LS and HS forms of the above three compounds were taken from X-ray experiments $^{23,29,30}$ and were assumed to be fixed (frozen). The space groups of the crystals are $P b c n, P b c n$ and $C 2 / c$ respectively; the number of molecules in a unit cell, $Z=4$, in all three cases. Further details of the crystal structures and the intermolecular contacts can be found in ref. 1 . The validity of this "rigid-body" approximation can be tested and the analysis of the difference of the vibrational parameters for a crystal formed by different spin isomers demonstrated ${ }^{31,32}$ that the non-rigidity is relatively small for both HS and LS forms. In other words, one can imagine a mixed crystal as one formed by the molecules with two different structures (LS and HS), but not with intermediate ones. The geometrical change of an individual molecule (the spin transition itself) takes place instantaneously on the thermodynamical time scale.

To study how the concentrations of the spin isomers and their distribution in the crystal affect the energy (enthalpy) of a mixed crystal, we performed minimization of the enthalpies of various mixed crystals as a function of the unit cell parameters, positions of the centers of masses (CM), and the rotation angles of the molecules in the unit cell. In each case the concentrations of the HS molecules and their distribution in the crystal were kept constant. The mixed crystals to be included in the consideration were built as follows. In the crystals formed by repetition of the original unit cells, containing four molecules each, total number of possible distributions of the spin forms equals to $2^{4}=16$. Due to the symmetry only seven of them are different: one for $x=0$; one (out of four) for $x=0.25$; three (out of six) for $x=0.5$; one (out of four) for $x=0.75$; and one for $x=1$. Seven data points do not suffice to reliably fit a model with three free parameters $(C, D$ and $\Gamma)$, in particular if we take into consideration that such a dataset includes only highly correlated patterns of spin distribution (due to symmetry) and are hardly extendable to nontrivial spin distributions. The simplest generalization is to include in the consideration the mixed crystals formed by repetition of doubled unit cells. In this case the total number of distributions of the spin forms in the crystal reaches $2^{8} \cdot 3=768$ (the factor of 3 appears since the duplication of a unit cell is possible along either of $a, b$, or $c$ directions). Only some part of these 768 distributions represent different crystal structures (respectively, 92 mutually different structures in the cases of $\mathrm{Fe}(\mathrm{phen})_{2^{-}}$ $(\mathrm{NCS})_{2}$ and $\mathrm{Fe}(\mathrm{btz})_{2}(\mathrm{NCS})_{2}$ and 96 in the case of $\mathrm{Fe}(\mathrm{bpz})_{2}($ bipy)). This amount of data fairly suffices for a least-squares treatment.

The crystal enthalpy was minimized as a function of 51 parameters $(a, b, c$, three unit cell angles, three rotation angles for each of eight molecules in the doubled unit cell, three CM position coordinates for seven out of eight molecules in the unit cell). Pressure was set to be $1 \mathrm{~atm}$. Arithmetic mean values of the parameters of the pure LS and HS crystal were taken as initial approximations. The minimization was performed in three stages. First, we minimized the energy calculated according to eqn (3) with only one layer of unit cells adjacent to that with the lattice vector $\mathbf{0}$. By this fast estimates of the approximate location of the minima are made. After that, we corrected the location of the minima, taking into consideration one more layer of adjacent unit cells. Finally, we computed the energy (without performing a new minimization) for the crystal parameters found at the previous stage, accounting for in total four layers of unit cells adjacent to the given one (those $\mathbf{r}=\left(r_{a}, r_{b}, r_{c}\right)$, for which $\left|r_{a}\right|,\left|r_{b}\right|,\left|r_{c}\right| \leq 4$ were included). This algorithm of the enthalpy minimization is based on the results of ref. 1 where it was shown that even one layer of adjacent unit cells yields the optimal lattice parameters within $0.1 \%$ of the optimal parameters obtained with a larger number of adjacent layers. At the same time, the energy (or the enthalpy) becomes stable (the difference is less than $0.01 \mathrm{kcal} \mathrm{mol}^{-1}$ ) only when at least three layers of adjacent unit cells are included. This procedure was applied to each mixed crystal and for each of the three potentials. As a result, we obtained the enthalpies of each mixed crystal as functions of the concentration $x$ of the HS molecules and their distribution in the crystal.

The experimental data concerning the $x(T)$ curves and the "experimental" values of the $\Gamma$ parameter, as extracted from these curves, call for a separate discussion. Since the HS forms of the studied Fe(II) complexes have 4 unpaired electrons, while in the LS molecules all electrons are paired, a change of the $x$ value manifests itself in a change of the magnetic susceptibility $\chi$ of the crystal. According to the Curie law, the product of the molar magnetic susceptibility $\chi_{M}$ and the absolute temperature $T$ should be constant for a given paramagnetic material. A number of authors, however, simplify the situation by concluding that the $x(T)$ values can be calculated by dividing the $\chi_{\mathrm{M}} T$ value at a given temperature by the $\chi_{\mathrm{M}} T$ value at the highest temperature for which they made measurements (for example, see ref. 23, 29, 30 and 33). This approach is not adequate due to the following reasons.

- The LS form has a non-zero magnetic susceptibility, despite the fact that all electrons are paired. ${ }^{34}$ The estimated molar susceptibility $\chi_{\mathrm{LS}}=2.07 \times 10^{-4} \mathrm{~cm}^{3} \mathrm{~mol}^{-1} \cdot 34$

- For the HS form the effective magnetic moment $n_{\text {eff, }}$ and therefore the value of $\chi_{\mathrm{M}} T$, deviates from the Curie law due to spin-orbit coupling. ${ }^{34}$ Namely, in CGS units, $\chi_{\mathrm{M}} T=$ $0.125 \mathrm{~cm}^{3} \mathrm{~K} \mathrm{~mol}^{-1} \cdot n_{\mathrm{eff}}^{2}$, and

$$
\begin{aligned}
& n_{\mathrm{eff}}^{2} \\
& =\frac{(73.5-40.5 \theta) e^{-3 / \theta}+(67.5+12.5 \theta) e^{-1 / \theta}+(84+28 \theta) e^{2 / \theta}}{3 e^{-3 / \theta}+5 e^{-1 / \theta}+7 e^{2 / \theta}},
\end{aligned}
$$

where $\theta=-4 k T / \zeta$, with the spin-orbit constant for $\mathrm{Fe}(\mathrm{II})$ $\zeta=410 \mathrm{~cm}^{-1}$.

- The high-temperature limit of the $\chi_{\mathrm{M}} T$ value was not accurately studied. All experimental measurements were performed below $293 \mathrm{~K}$. At least in the case of the $\mathrm{Fe}(\mathrm{btz})_{2}(\mathrm{NCS})_{2}$ compound, it is evident (see Fig. 1 in ref. 30) that the $\chi_{\mathrm{M}} T$ value at this temperature is still far from its high-temperature limit. In addition, the limiting value of $x$ at high temperatures is not mandatorily equal to unity (for example, in the SDM, $\left.\lim x(T \rightarrow \infty)=(1+\exp (-\Delta S / R))^{-1}<1\right)$. As a result, it is not clear how many LS molecules are left in the crystals in 
each of these three cases at $290 \mathrm{~K}$, and how to calculate their amount at intermediate temperatures.

- There are evidences that the thermodynamical equilibrium is probably not reached in the systems (at least, in the $\mathrm{Fe}(\text { phen })_{2}(\mathrm{NCS})_{2}$ and $\mathrm{Fe}(\mathrm{btz})_{2}(\mathrm{NCS})_{2}$ crystals $)$ while the measurements of magnetic susceptibility are performed, especially at low temperatures. This is pointed out by discrepancies of the experimental $\chi_{\mathrm{M}} T v s$. $T$ curves for the samples prepared by different techniques, or measured in different groups. ${ }^{29,30,35}$ Mössbauer spectroscopy confirms the conclusion about incompleteness of the spin transition in some samples at low temperatures. $^{35}$

Taking these issues into consideration, we estimated the "experimental" $\Gamma$ parameters of the SDM of the materials at hand as follows. Assuming the magnetic susceptibility of a mixture of LS and HS forms to linearly depend on their concentrations in the sample, we have:

$$
\left(\chi_{\mathrm{M}} T\right)^{\mathrm{SDM}}=(1-x(T)) \chi_{\mathrm{LS}} T+x(T) \cdot 0.125 \mathrm{~cm}^{3} \mathrm{~K} \mathrm{~mol}^{-1} \cdot n_{\mathrm{eff}}^{2}(T, \zeta) .
$$

The $\Delta H, \Delta S$ and $\Gamma$ parameters of the SDM can be found from the experimental data on $\chi_{\mathrm{M}} T v s . T$ by the (nonlinear) least squares method, minimizing numerically the sum

$$
\sum_{T}\left(\left(\chi_{\mathrm{M}} T\right)^{\mathrm{SDM}}-\left(\chi_{\mathrm{M}} T\right)^{\exp }\right)^{2}
$$

taken in all points of temperature for which the experimental data are available, ${ }^{23,29,30}$ with respect to $\Delta H, \Delta S$, and $\Gamma$. Here $\left(\chi_{\mathrm{M}} T\right)^{\exp }$ are the experimental values for given $T$, and $\left(\chi_{\mathrm{M}} T\right)^{\mathrm{SDM}}$ are calculated by eqn (7) from the $x(T)$ values predicted by the SDM with the given $\Delta H, \Delta S$, and $\Gamma$. The results are given in Table 1. Taking into consideration that the experimental data at low temperatures probably refer to nonequilibrium states, we complemented the results of minimization of the sum of squares in the entire available temperature intervals by the results of minimization for the experimental data in the ranges of $168-210 \mathrm{~K}\left(\mathrm{Fe}(\text { phen })_{2}(\mathrm{NCS})_{2}\right), 170-290 \mathrm{~K}$ $\left(\mathrm{Fe}(\mathrm{btz})_{2}(\mathrm{NCS})_{2}\right)$, and $129-229 \mathrm{~K}\left(\mathrm{Fe}(\mathrm{bpz})_{2}\right.$ (bipy)). Since the values of $\chi_{\mathrm{M}} T$ change rapidly with $T$ in these intervals, the system is more likely to be able to come to the equilibrium. The values of the $\Delta H, \Delta S$, and $\Gamma$ parameters, calculated by these two approaches (those obtained from the restricted temperature intervals are given in brackets), are rather close to each other (except for the $\Delta H$ value for the $\mathrm{Fe}(\mathrm{btz})_{2}(\mathrm{NCS})_{2}$ crystal). The deviations of the predicted $\chi_{\mathrm{M}} T$ from the experimental ones are rather small at the intermediate and high temperatures (0.07- $0.10 \mathrm{~cm}^{3} \mathrm{~K} \mathrm{~mol}^{-1}$ for all three compounds, in comparison to the magnitude of variation of $\chi_{\mathrm{M}} T$ itself $\left.\sim 3 \mathrm{~cm}^{3} \mathrm{~K} \mathrm{~mol}^{-1}\right)$, but significantly increase at low temperatures $\left(\sim 0.5 \mathrm{~cm}^{3} \mathrm{~K} \mathrm{~mol}^{-1}\right.$ for the $\mathrm{Fe}(\text { phen })_{2}(\mathrm{NCS})_{2}$ and $\mathrm{Fe}(\mathrm{btz})_{2}(\mathrm{NCS})_{2}$ crystals, which corresponds to the leftover concentration of the HS molecules as high as $15-18 \%$ ).

The results reported for "sample A" of the $\mathrm{Fe}(\text { phen })_{2}(\mathrm{NCS})_{2}$ compound in ref. 35 suggest that the equilibrium was reached for that sample at low temperatures, and no HS molecules in the sample were found at $4.2 \mathrm{~K}$ by Mössbauer spectroscopy (in contrast to "sample B" in the same publication). However, the SCT in "sample A" was so abrupt that our attempts to estimate $\Delta H$ and $\Gamma$ values from the $x(T)$ plot (Fig. 4 in ref. 35) did not lead to stable results. For this reason, we will be using the above values of $\Delta H$ and $\Gamma$, derived from the experimental data from ref. 29 , but will use the data from ref. 35 in evaluation of the results (see Fig. 2).

The values of the $\Gamma$ parameter given in Table 1 are below referred to as the "experimental" ones to be compared with the results of modeling by the atom-atom potential method.

\section{Results and discussion}

Using the sets of enthalpies calculated for different mixed crystals of the given compound by the method described in the previous section, one can apply the least squares procedure to estimate the phenomenological parameters of a macroscopic model. If for a given $x$ all configurations of the HS molecules in a crystal are taken as equiprobable, the parameter $\Gamma$ of the Slichter-Drickamer model can be estimated from the regression

$$
H=C+D x+\Gamma x(1-x),
$$

where $H$ is the enthalpy of the mixed crystal relative to the enthalpy of the pure LS crystal, which thus yields microscopic "mean-field" estimates of the parameters of the SDM as derived from the atom-atom potentials.

On the other hand, the configurations of the HS molecules with a relatively high energy (as compared to other configurations for a given $x$ ) will occur less frequently in the Gibbs ensemble. To get a simplest account for this effect, we estimated the parameters in the following approximation:

$$
\min H(x)=C^{\prime}+D^{\prime} x+\Gamma^{\prime} x(1-x),
$$

where $\min H(x)$ is the minimum enthalpy among the enthalpies of all configurations with the given $x$ value. This will be termed as microscopic "overcorrelated" estimates of the parameters of the SDM as derived from the atom-atom potentials.

It is important to verify that the results of such treatment are independent of the specific atom-atom potential used for modeling the mixed crystals, especially in view of that experimental data on the energies or enthalpies of the mixed crystals formed by both LS and HS isomers are currently absent. This analysis shows that the enthalpy estimates coming from different potentials are consistent with each other,

Table 1 Empirical values of the parameters of the Slicher-Drickamer model obtained by the nonlinear least squares method. The values in parenthesis refer to the restricted temperature intervals (see details in the text).

\begin{tabular}{llll}
\hline Compound & $\Delta H, \mathrm{kcal} \mathrm{mol}^{-1}$ & $T_{c}^{\mathrm{exp}}=\Delta H / \Delta S, \mathrm{~K}$ & $\Gamma^{\mathrm{exp}}, \mathrm{kcal} \mathrm{mol}^{-1}$ \\
\hline $\mathrm{Fe}(\mathrm{phen})_{2}(\mathrm{NCS})_{2}$ & $0.8(0.9)$ & $177.8(177.8)$ & $0.710(0.715)$ \\
$\mathrm{Fe}(\mathrm{btz})_{2}(\mathrm{NCS})_{2}$ & $1.1(1.9)$ & $231.7(233.7)$ & $0.47(0.30)$ \\
$\mathrm{Fe}(\mathrm{bpz})_{2}(\mathrm{bipy})$ & $1.1(1.2)$ & $163.2(163.3)$ & $0.55(0.54)$ \\
\hline
\end{tabular}




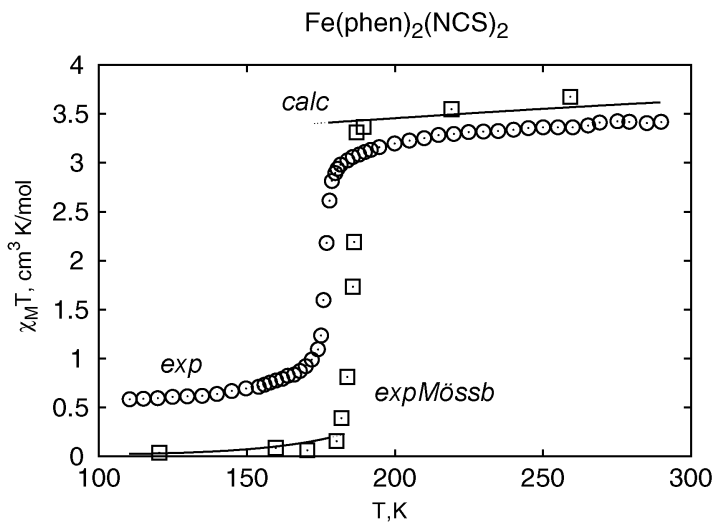

$\mathrm{Fe}(\mathrm{btz})_{2}(\mathrm{NCS})_{2}$

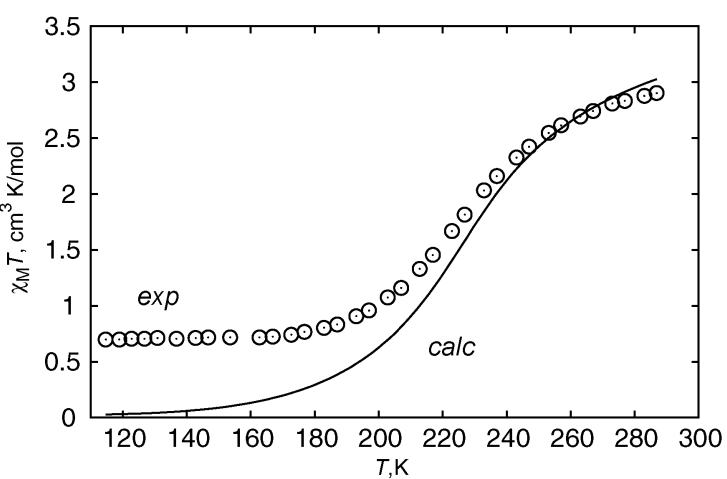

$\mathrm{Fe}(\mathrm{bpz})_{2}(\mathrm{bipy})$

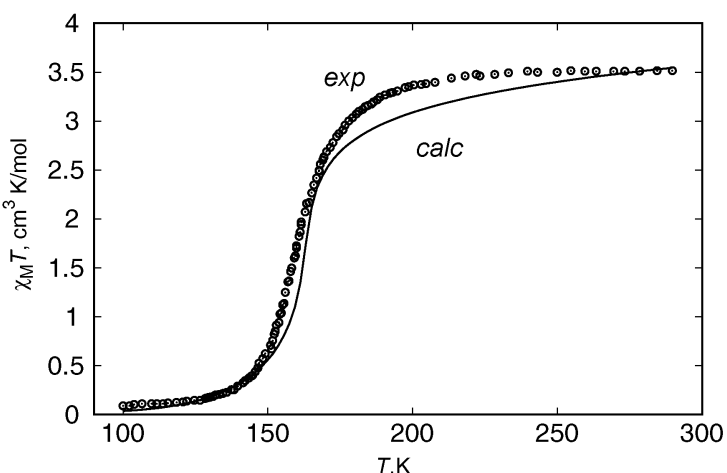

Fig. 2 Comparison of the $\chi_{\mathrm{M}} T$ vs. $T$ curves measured experimentally ("exp") $23,29,30$ and those calculated in this work ("calc"). For the $\mathrm{Fe}(\text { phen })_{2}(\mathrm{NCS})_{2}$ compound, the experimental cooling mode is shown; the hysteresis loop does not exceed $1 \mathrm{~K}$. For the two other substances, no hysteresis was experimentally detected. Calculations were made with the Slichter-Drickamer model, using the $\Gamma$ values taken from Table 2 ( $\Gamma^{\prime}, 6$-expM parameterization), and the $\Delta H$ and $T_{\mathrm{c}}$ values from Table 1. For the Fe(phen $)_{2}(\mathrm{NCS})_{2}$ compound, the experimental data for "sample A" from ref. 35 are also shown ("expMössb"); the values of $x$ translated into values of $\chi_{M} T$ by eqn (7).

differing typically by $0.2-0.6 \mathrm{kcal} \mathrm{mol}^{-1}$. The major fraction of the difference can be attributed to a systematic error, presumably arising from the uncertainty in the energy scale (see more detailed discussion in the Appendix).

As mentioned in previous section, the mixed crystals with four or eight molecules per unit cell were included in the sample; respectively, the fraction of HS molecules in a crystal $x$ for different data points was equal to: $0,1 / 8=12.5 \%$,
$2 / 8=25 \%, \ldots, 7 / 8=87.5 \%, 8 / 8=100 \%$. When estimating the optimal values of $C, D$ and $\Gamma$ in the "mean-field" approximation (9), we used the weighted least squares method to account for the fact that different number of structures in the sample represent different number of the values of $x$ (for example, $x=0$ is respresented by one structure, $x=0.125$ - by three structures: one with a unit cell with $Z=8$ obtained by doubling of an original unit cell with $Z=4$

Table 2 The parameters of the simplified approximations eqn (9) and (10) for the Fe(phen $)_{2}(\mathrm{NCS})_{2}, \mathrm{Fe}(\mathrm{btz})_{2}(\mathrm{NCS})_{2}$ and Fe(bpz $)_{2}(\mathrm{bipy})$ compounds found by the method of least squares. For each model the value of $R^{2}$ and the mean-square error of the approximation $(\sigma)$ are reported. Standard errors are given in parenthesis after the estimations of the corresponding parameters (errors are measured in the units of the last digit of the parameter). All values (except for $R^{2}$ ) are in $\mathrm{kcal} \mathrm{mol}^{-1}$.

\begin{tabular}{|c|c|c|c|c|c|c|c|c|}
\hline \multirow{2}{*}{$\begin{array}{l}\text { Compound } \\
\text { Potential }\end{array}$} & \multicolumn{3}{|c|}{$\mathrm{Fe}(\text { phen })_{2}(\mathrm{NCS})_{2}$} & \multicolumn{3}{|c|}{$\mathrm{Fe}(\mathrm{btz})_{2}(\mathrm{NCS})_{2}$} & \multicolumn{2}{|c|}{$\mathrm{Fe}(\mathrm{bpz})_{2}($ bipy $)$} \\
\hline & 6-expM & 6-exp & $6-12$ & 6-expM & 6-exp & $6-12$ & 6-exp & $6-12$ \\
\hline \multicolumn{9}{|c|}{ The "mean-field" SDM eqn (9): } \\
\hline$C$ & $0.017(16)$ & $0.016(15)$ & $0.016(15)$ & $0.005(8)$ & $0.010(9)$ & $0.015(9)$ & $0.021(10)$ & $0.040(14)$ \\
\hline$D$ & $1.50(2)$ & $1.20(2)$ & $1.78(2)$ & $-1.07(1)$ & $-1.11(1)$ & $-0.19(1)$ & $0.43(1)$ & $1.12(2)$ \\
\hline$\Gamma$ & $2.06(7)$ & $1.94(7)$ & $1.93(7)$ & $1.06(4)$ & $1.44(4)$ & $1.69(4)$ & $1.38(4)$ & $2.02(6)$ \\
\hline$R^{2}$ & 0.987 & 0.981 & 0.991 & 0.992 & 0.992 & 0.957 & 0.952 & 0.978 \\
\hline$\sigma$ & 0.061 & 0.059 & 0.058 & 0.032 & 0.035 & 0.036 & 0.043 & 0.062 \\
\hline \multicolumn{9}{|c|}{ The "overcorrelated" SDM eqn (10): } \\
\hline$C^{\prime}$ & $0.035(40)$ & $0.029(35)$ & $0.028(31)$ & $0.019(27)$ & $0.029(35)$ & $0.035(37)$ & $0.040(38)$ & $0.067(58)$ \\
\hline$D^{\prime}$ & $1.52(5)$ & $1.21(4)$ & $1.79(4)$ & $-1.07(3)$ & $-1.11(4)$ & $-0.19(5)$ & $0.45(5)$ & $1.15(7)$ \\
\hline$\Gamma^{\prime}$ & $1.19(18)$ & $1.21(16)$ & $1.29(14)$ & $0.56(12)$ & $0.83(16)$ & $1.09(16)$ & $0.59(17)$ & $0.88(26)$ \\
\hline$R^{2}$ & 0.994 & 0.993 & 0.997 & 0.994 & 0.991 & 0.910 & 0.941 & 0.977 \\
\hline$\sigma$ & 0.050 & 0.043 & 0.038 & 0.033 & 0.043 & 0.045 & 0.047 & 0.071 \\
\hline
\end{tabular}


along the $x$ axis; another with a unit cell with $Z=8$ obtained by doubling along the $y$ axis; and the third - by doubling along the $z$ axis). The system of weights was developed in such a way that all the values of $x$ in the range from 0 to 1 are uniformly represented. The maximal weight (1) was assigned to the pure LS and pure HS configurations; the minimum weight (0.019) was attributed to several structures with $x=0.5$. As for the case of the "overcorrelated" approximation (10), the system of weights is not needed there, since by definition of $\min H(x)$ every value of $x$ in the range from 0 to 1 is represented by only one mixed crystal and therefore by only one data point.

The resulting effective parameters and the statistical characteristics of the two models are given in Table 2. All regressions have the standard errors as low as $0.03-0.07 \mathrm{kcal} \mathrm{mol}^{-1}$, which does not exceed the presumable error of the atom-atom scheme and is much less than the value of $k_{\mathrm{B}} T$ at the temperatures characteristic for the SCT. As one could expect, the values of $\Gamma^{\prime}$ for the regression eqn (10) are always smaller than the corresponding values of $\Gamma$ for the regression eqn (9), since the energy in the correlated approximation is always lower than the mean-field ones for all intermediate values of $x$, but is the same for the pure LS and HS crystals. The deviation of $\min H(x)$ from the linear function is smaller than that of $H(x)$, and thus the effective value of $\Gamma^{\prime}$ is smaller than that of $\Gamma$ as well.

The true value of $\Gamma$ will lie between the two extreme estimates, since at any finite temperature all possible configurations of the HS molecules in the crystal occur in the Gibbs ensemble. The exact value of the parameter may be obtained by explicit Monte-Carlo simulations of the ensemble or more accurately approximated, e.g., by the quasichemical method. ${ }^{36}$

The standard error analysis shows that the simplest "mean-field" eqn (9) and "overcorrelated" eqn (10) approximations, are quite acceptable, in spite of their simplicity. The comparison of the calculated $\Gamma$ values with the experimental values (see Table 1) shows the following. The signs and the orders of magnitudes of the $\Gamma$ parameters were predicted correctly in all three cases, as well as the relations between these parameters for the different materials: $\Gamma\left(\mathrm{Fe}(\mathrm{phen})_{2}(\mathrm{NCS})_{2}\right)>\Gamma\left(\mathrm{Fe}(\mathrm{bpz})_{2^{-}}\right.$ (bipy) $)>\Gamma\left(\mathrm{Fe}(\mathrm{btz})_{2}(\mathrm{NCS})_{2}\right) ; \Gamma^{\prime}\left(\mathrm{Fe}(\text { phen })_{2}(\mathrm{NCS})_{2}\right)>\Gamma^{\prime}\left(\mathrm{Fe}(\mathrm{bpz})_{2}-\right.$ (bipy) $)>\Gamma^{\prime}\left(\mathrm{Fe}(\mathrm{btz})_{2}(\mathrm{NCS})_{2}\right)$ as in the "mean-field" likely in the "overcorrelated" approximations. The estimated parameters are all positive and thus correspond to "cooperative" behavior. The absolute "mean-field" values are overestimated by a factor of two. Partially, this may be caused by the fact that we considered only the mixed crystals with unit cells involving four or eight molecules, and thus could underestimate possible relaxations (especially elastic) in the crystal and therefore overestimate the effective values of the $\Gamma$ parameters. Another possible source of the error, which in our opinion is the major source (see, however, below), is the uncertainty in the absolute scale of the energy as calculated by the atom-atom potentials method, as it is discussed in the Appendix. To verify this suggestion, independent (preferably experimental) studies of the intermolecular interaction energies are required.

The "overcorrelated" SDM parameters $\Gamma^{\prime}$ show much better agreement with experiment. The "mean-field" parameters $\Gamma$ in all three cases satisfy the condition $\Gamma>\Gamma_{\mathrm{c}}$ thus suggesting the first-order SCT which must be abrupt and eventually manifest a thermal hysteresis. Using the "overcorrelated" parameters $\Gamma^{\prime}$ significantly improves qualitative agreement with experiment in this respect particularly if the modified parameterization 6-expM is applied. The SDM with thus defined parameters predicts $\mathrm{Fe}(\mathrm{phen})_{2}(\mathrm{NCS})_{2}$ to manifest an abrupt SCT with hysteresis $\left(\Gamma^{\prime}>\Gamma_{\mathrm{c}}\right)$ in agreement with experiment (though the experimental width of the hysteresis loop does not exceed $1 \mathrm{~K}$ ); for $\mathrm{Fe}(\mathrm{btz})_{2}(\mathrm{NCS})_{2}$ a gradual "cooperative" SCT without hysteresis must be expected $\left(\Gamma^{\prime}<\Gamma_{\mathrm{c}}\right)$, also in agreement with experiment; finally, for $\mathrm{Fe}(\mathrm{bpz})_{2}$ (bipy) a sharper SCT without hysteresis may be expected, which corresponds to the experiment $\left(\Gamma^{\prime}\right.$ is slightly less than $\Gamma_{\mathrm{c}}$ with the 6-exp potential, though the opposite relation is obtained with the 6-12 potential). This comparison is illustrated in Fig. 2. As one can see, a semiquantitative agreement was reached. It is especially impressive that the curve predicted for the $\mathrm{Fe}(\mathrm{phen})_{2}(\mathrm{NCS})_{2}$ compound ("calc") with the use of $\Delta H$ value found from experimental data for one sample ("exp"), where equilibrium was presumably not reached at low temperatures, is in excellent agreement with the experimental data for another sample ("expMössb") measured, presumably, in equilibrium.

\section{Conclusion}

In the present paper we applied for the first time the method of atom-atom potentials to mixed crystals formed by high-spin and low-spin forms of the $\mathrm{Fe}(\mathrm{II})$ complexes capable of undergoing SCT, in order to check the adequacy of the interaction energy treatment with use of the Slichter-Drickamer model, and to obtain independent theoretical estimates of the corresponding phenomenological parameters.

The approximation of a mixed crystal enthalpy by the second order polynom in the concentration $x$ of the HS molecules in the crystal (Table 2), neglecting the details of the spatial distribution of HS molecules, already provides a good level of precision (standard errors of $0.03-0.07 \mathrm{kcal} \mathrm{mol}^{-1}$ ) in comparison to the $k_{\mathrm{B}} T$ value (typically in the range of 0.3-0.6 kcal mol ${ }^{-1}$ ) and to the typical discrepancies of the enthalpies of the same crystal estimated with the different atom-atom potentials (standard errors up to $0.6 \mathrm{kcal} \mathrm{mol}^{-1}$ ). This approximation is central to the Slichter-Drickamer model, though it has not been verified up to now.

The values of the $\Gamma$ parameters of the Slichter-Drickamer model as estimated in the "mean-field" approximation were found to be 1.9-2.1, 1.0-1.7, and 1.4-2.0 kcal mol ${ }^{-1}$ for the $\mathrm{Fe}(\text { phen })_{2}(\mathrm{NCS})_{2}, \mathrm{Fe}(\mathrm{btz})_{2}(\mathrm{NCS})_{2}$ and $\mathrm{Fe}(\mathrm{bpz})_{2}(\mathrm{bipy})$ compounds respectively, depending on the parameterization used (the "experimental" values being $0.71,0.3-0.5$ and $\left.0.55 \mathrm{kcal} \mathrm{mol}^{-1}\right)$. It is remarkable that the discrepancies between the different atom-atom potentials used for the same substance were mainly absorbed by the $D$ parameters of the model, while the $\Gamma$ parameters are less sensitive to them. Nevertheless, the "mean-field" estimates of the SDM parameter $\Gamma$ turn out to be several times larger than the "experimental" estimates. By contrast, we could demonstrate that correlations in the distribution of the spin-isomers in the crystal are crucial for a correct description of the SCT. Within the "overcorrelated" model a semiquantitative agreement of the calculated values $\Gamma^{\prime}$ with the "experimental" ones is shown to be possible. 
Although even the simple "mean-field" model leads to correct predictions of the sign of the $\Gamma$ SDM-parameters and their ratios for different compounds, semiquantitavely correct performance of the "overcorrelated" model suggests that the local correlations in the distribution of spin-isomers play the leading role in building the correct picture of the phenomenon under study. Taking the correlations into account even in a simplistic way, we could reproduce qualitatively different behavior the three materials under consideration exhibit under the SCT. Specifically, the crystals of $\mathrm{Fe}(\text { phen })_{2}(\mathrm{NCS})_{2}$ manifest an abrupt SCT with hysteresis, the $\mathrm{Fe}(\mathrm{btz})_{2}(\mathrm{NCS})_{2}$ compound shows a gradual SCT without hysteresis, and, finally, in the case of $\mathrm{Fe}(\mathrm{bpz})_{2}$ (bipy) a sharper SCT without hysteresis is observed. Using the "overcorrelated" estimate for the $\Gamma^{\prime}$ SDM-parameters, we for the first time could successfully predict the type of the SCT in these various crystals, based on the microscopic considerations. This result is important not only for theoretical study of this phenomenon, but also for practical applications, opening a way for rational design of a wide spectrum of industrially applicable SCT materials with specific desired properties.

The above agreement with experiment obtained with use of the atom-atom model of the van der Waals interactions between the molecules in different spin states in the crystals supports an old hypothesis ${ }^{4}$ of one of us concerning the origin of the cooperative interactions responsible for appearance of the phenomenological parameter $\Gamma$. Basically it is the difference between the interaction energies of the spin isomers

$$
\Gamma=\frac{1}{2} z \varepsilon ; \varepsilon=E_{\mathrm{HL}}-\frac{1}{2}\left(E_{\mathrm{HH}}+E_{\mathrm{LL}}\right)
$$

whatever their origin is ( $z$ is the number of nearest neighbors). It suggests that at least in the case of neutral molecules considered here the only reason for existence of the required energy differences is the difference of "sizes" of the HS and LS spin isomers accompanied by the lattice relaxations as proposed yet in ref. 2. In a way it means that any specific intramolecular mechanism is superfluous for explaining the cooperative behavior in the SCT since that latter is of purely intermolecular origin.

It is instructive to compare these arguments with the approach taken in ref. 37. In that work the Coulomb interactions between the charge distributions in a spin-active model molecule with counteranion charges provided specific intramolecular mechanism responsible for the hysteresis in the SCT. We incline to the idea that the hysteresis in SCT appears (whenever it happens) as a consequence of cooperativity, leading to the first order phase transition in the system irrespective to the origin of the interactions yielding cooperativity. Thus we consider possible contribution of the ref. 37 mechanism to cooperativity. Their approach is based on a high-quality ab initio calculation performed, however, for an oversimplified model system formed by charged particles $\left[\mathrm{Fe}(\mathrm{NCH})_{6}\right]^{2+}$ as assembled in a lattice together with $\mathrm{Cl}^{-}$counterions. The authors of ref. 37 ascribe the hysteresis to an enormous charge transfer (of $c a .0 .5 \bar{e}$ ) allegedly occurring throughout the SCT. We have to admit, that in an ionic system as that studied in ref. 37 one cannot a priori exclude an electrostatic contribution to the intermolecular interactions proportional to the charge transfer in case it occurs. Indeed, the charge transfer affects significantly the hexadecupole (16-pole) momentum of the model $\left[\mathrm{Fe}(\mathrm{NCH})_{6}\right]^{2+}$ ion (all lower momenta are vanishing due to symmetry in either spin form) and thus their interactions with the counterions, which decay as $R^{-5}$, as well as those between the HS and LS forms of the model ion, which decay, however, as $R^{-9}$-faster than the van der Waals forces - and thus should not significantly contribute to the intermolecular interactions. Applying reasoning described in details in ref. 38 we estimate the electrostatic contribution to the above $\varepsilon$ parameter to be of the order of magnitude

$$
\varepsilon \sim \frac{(\Delta h)^{2}}{R^{9}}
$$

where $\Delta h$ is the variation of the hexadecupole momentum of a $\left[\mathrm{Fe}(\mathrm{NCH})_{6}\right]^{2+}$ ion under spin transition and $R$ is the distance between the centers of hexadecupoles - in our case between the $\mathrm{Fe}$ atoms. The transfer of $0.5 \overline{\mathrm{e}}$ on $\delta r \approx 2 \AA$ results in the variation of the hexadecupole momentum $\sim \delta r^{4} \approx 128$ a.u. Combining this with the interatomic separation of $c a .20$ a.u. we find the electrostatic contribution to $\varepsilon$ to be of the order of $0.01 \mathrm{~K}$. That allows us to conclude that the amount of the electrostatic contribution as estimated in ref. 37 (ca. $720 \mathrm{~K}-\mathrm{a}$ huge overestimate as compared to experimental values and our results) is largely coming from the hexadecupole-counterion interactions. Even if the charge transfers required for the electrostatic mechanism had taken place (see below) their effect in the crystals formed by electroneutral molecules - as those studied in the present paper-must be even smaller since in this case the first nonvanishing contribution to the energy would be the hexadecupole-dipole one as decaying as $R^{-6}$, rather than $R^{-5}$, and thus giving smaller contribution. We dare to suppose, however, that the changes of the $\mathrm{Fe}$ atomic charges upon the SCT are overestimated in ref. 37. This view is supported by the problems with describing the excitation spectrum of the $\left[\mathrm{Fe}(\mathrm{NCH})_{6}\right]^{2+}$ ion reported in the same paper. By contrast, our earlier estimates, ${ }^{38}$ which are in perfect agreement with the spectra, demonstrate that the charge transfer under an SCT does not exceed 0.02e and that the contribution of electrostatic forces into the $\Gamma$ parameter for realistic low-symmetry molecular models does not exceed $\sim 1 \%$ of its measured magnitude. On the other hand, if the charge transfer does not take place during the SCT, the standard atom-atom potentials used in the present study effectively take into account all the charges occurring in the organic ligands, which ultimately contribute to the energy of molecular crystals as effective higher multipoles.

\section{Appendix. Comparative analysis of the enthalpies calculated with the use of various versions of the atom-atom potentials}

In the case of the $\mathrm{Fe}(\mathrm{phen})_{2}(\mathrm{NCS})_{2}$ mixed crystals, the enthalpies estimated with use of the atom-atom potentials 6-expM, 6-exp and 6-12, are fairly close to each other. The corresponding correlation coefficients and mean-square deviations are given in Table 3. These deviations can serve as estimates of the uncertainties introduced into the model of 
Table 3 Consistency of the enthalpies of the mixed crystals formed by various spin forms of the $\mathrm{Fe}(\mathrm{phen})_{2}(\mathrm{NCS})_{2}$ compound as calculated with use of various atom-atom potentials

\begin{tabular}{llllllll}
\hline & \multicolumn{2}{c}{ Correlation coefficient } & & \multicolumn{3}{l}{ Mean-square difference, $\mathrm{kcal} \mathrm{mol}^{-1}$} \\
& 6-expM & 6-exp & 6-12 & & 6-expM & 6-exp & 6-12 \\
\hline 6-expM & 1 & 0.996 & 0.991 & & 0.20 & 0.15 \\
6-exp & 1 & 0.984 & & 0 & 0.35 \\
6-12 & & & 1 & & & 0 \\
\hline
\end{tabular}

SCT by the choice of a specific atom-atom parameterization (as opposed to, for example, the uncertainties arising from the assumptions of the Slichter-Drickamer model, or from the lack of equilibration of samples in experiments). The similarity of two spin forms allows one to calculate the relative energy of the mixed crystals (that is, the energy of a mixed crystal relative to the energy of the pure LS or HS crystal), regardless of the parametrization system used, with the precision of ca. $0.2 \mathrm{kcal} \mathrm{mol}^{-1}$, which is much smaller than the error in the calculated absolute values of energies (the energies counted off the level of infinitely separated molecules) of the same crystals (typically ca. $1 \mathrm{kcal} \mathrm{mol}^{-1}$, see ref. 1). The main reason of the remaining discrepancy is a systematic error. The mean-square value of the difference $H_{6 \text {-exp }}-k H_{6-\operatorname{expM}}$ is minimized with $k=0.848$ and equals to only $0.026 \mathrm{kcal} \mathrm{mol}^{-1}$ (7.3 times less than the mean-square value of $H_{6-\exp }-H_{6-\operatorname{expM}}$ ), and the minimum mean-square value of $H_{6-12}-k H_{6-\operatorname{expM}}$ is achieved for $k=1.096$ and equals to $0.051 \mathrm{kcal} \mathrm{mol}^{-1}$ (2.5 times less than the mean-square value of $\left.H_{6-12}-H_{6 \text {-expM }}\right)$. These estimates are justified from the physical viewpoint by lack of experimental data on crystal energies (in contrast with data on the geometry of equilibrium lattice structures). Indeed, a proportional increase of all $A$ and $B$ parameters (or, which is the same, of all potential well depths) in eqn (4) or (5) would only change the scale of energy, but not the position of minima on potential energy surfaces. Hence, the experimental X-ray structures alone do not suffice for determination of entire set of parameters, and they must be supplemented with the data with the dimension of energy (for example, sublimation heats). Typically, the data of the first type are much more numerous than those of the second type (for example, in ref. 26122 sublimation energies were used in comparison to thousands of data items-unit cell parameters $a, b, c, \alpha, \beta, \gamma, \mathrm{CM}$ positions and rotation angles of the molecules-extracted from the X-ray structures of 217 crystals). As a result, the optimal lattice parameters, as well as the ratio of the energy of one mixed crystal to the energy of another mixed crystal, can be predicted much better than the energies themselves. In case of the molecular crystals formed by the spin-active molecules, no experimental data on sublimation energies at all have been published so far. Although in ref. 1 we attempted to improve the parameterization of the atom-atom potentials method specially for better description of the geometry parameters of the $\mathrm{Fe}(\text { phen })_{2}(\mathrm{NCS})_{2}$ and $\mathrm{Fe}(\mathrm{btz})_{2}(\mathrm{NCS})_{2}$ crystals, ${ }^{28}$ we could not calibrate the method for the energies of these crystals. Therefore, it is reasonable to assume that the enthalpies of mixed crystals calculated with some parameterization system always contain systematic errors in the energy scale, unless special calibration against the experimental enthalpies is undertaken, and that these systematic errors will be different for different parameterization systems.

In the case of the $\mathrm{Fe}(\mathrm{btz})_{2}(\mathrm{NCS})_{2}$ mixed crystals, the agreement between the results obtained with use of the Lennard-Jones potential on the one hand and those coming from the Buckingham potentials on the other hand is much worse (see Table 4). However, the main source of the observed discrepancy is the systematic error as well, and the mean-square difference of the energies calculated with the different potentials can be decreased three times by the rescaling described in the previous paragraph-from $0.6 \mathrm{kcal} \mathrm{mol}^{-1}$ to $0.2 \mathrm{kcal} \mathrm{mol}^{-1}$.

Table 4 Consistency of the enthalpies of the mixed crystals formed by various spin forms of the $\mathrm{Fe}(\mathrm{btz})_{2}(\mathrm{NCS})_{2}$ compound as calculated with use of various atom-atom potentials

\begin{tabular}{llllllll}
\hline & \multicolumn{2}{c}{ Correlation coefficient } & & \multicolumn{2}{c}{ Mean-square difference, $\mathrm{kcal} \mathrm{mol}^{-1}$} \\
& 6-expM & 6-exp & $6-12$ & & 6-expM & 6-exp & 6-12 \\
\hline 6-expM & 1 & 0.995 & 0.661 & & 0.05 & 0.61 \\
6-exp & 1 & 0.713 & & 0 & 0.58 \\
6-12 & & 1 & & & 0 \\
\hline
\end{tabular}

Finally, in the case of the $\mathrm{Fe}(\mathrm{bpz})_{2}$ (bipy) mixed crystals, the correlation coefficient between the enthalpies found with the Lennard-Jones and Buckingham potentials equals to 0.947 , and the mean-square difference between them is $0.52 \mathrm{kcal} \mathrm{mol}^{-1}$. After linear elimination of the systematic error the mean-square difference decreases ten times (to $0.05 \mathrm{kcal} \mathrm{mol}^{-1}$ ). There is no difference between the modified Buckingham potential and the non-modified one for this substance.

To sum up, though the mean-square difference between the $\Delta H$ values estimated with different atom-atom potentials may reach $0.5-0.6 \mathrm{kcal} \mathrm{mol}^{-1}$, corrections for the systematic errors arising from uncertainty in the scale of $A$ and $B$ parameters decrease these discrepancies to $0.05-0.2 \mathrm{kcal} \mathrm{mol}^{-1}$.

\section{Acknowledgements}

This work has been partially supported by the RFBR through the grant No. 10-03-00155. ALT is grateful to Dr A. V. Soudackov for valuable discussion.

\section{References}

1 A. V. Sinitskiy, A. L. Tchougréeff, A. M. Tokmachev and R. Dronskowski, Phys. Chem. Chem. Phys., 2009, 11, 10983.

2 A. L. Tchougréeff, Mol. Cryst. Liq. Cryst., 1995, 274, 17; A. L. Tchougréeff and M. B. Darkhovskii, Int. J. Quantum Chem., 1996, 57, 903.

$3 \mathrm{Ph}$. Guionneau, M. Marchivie, G. Bravic, J.-F. Létard and D. Chasseau, Top. Curr. Chem., 2004, 234, 97.

4 A. L. Tchougréeff, Chem. Phys. Lett., 1993, 214, 627.

5 T. Kohlhaas, H. Spiering and P. Gütlich, Z. Phys. B: Condens. Matter, 1997, 102, 455.

6 H. Daubric, Ch. Cantin, C. Thomas, J. Kliava, J.-F. Létard and O. Kahn, Chem. Phys., 1999, 244, 75; S. Pillet, J. Hubsch and C. Lecomte, Eur. Phys. J. B, 2004, 38, 541.

7 H. Paulsen, L. Duelund, H. Winkler, H. Toftlund and A. X. Trautwein, Inorg. Chem., 2001, 40, 2201; S. Zein, G. S. Matouzenko and S. A. Borshch, Chem. Phys. Lett., 2004, 397, 475; G. S. Matouzenko, A. Bousseksou, S. A. Borshch, M. Perrin, S. Zein, L. Salmon, G. Molnar and S. Lecocq, Inorg. Chem., 2004, 43, 227; K. L. Ronayne, H. Paulsen, A. Höfer, A. C. Dennis, J. A. Wolny, A. I. Chumakov, V. Schünemann, H. Winkler, H. Spiering, A. Bousseksou, 
Ph. Gütlich, A. X. Trautwein and J. J. McGarvey, Phys. Chem. Chem. Phys., 2006, 8, 4685.

8 M. Reiher, Inorg. Chem., 2002, 41, 6928.

9 R. Boča and W. Linert, Monatsh. Chem., 2003, 134, 199.

$10 \mathrm{Ph}$. Gütlich and H. A. Goodwin, Top. Curr. Chem., 2004, 233, 1.

11 J.-F. Létard, Ph. Guionneau and L. Goux-Capes, Top. Curr. Chem., 2004, 235, 221.

12 H. Spiering, K. Boukheddaden, J. Linares and F. Varret, Phys. Rev. B: Condens. Matter Mater. Phys., 2004, 70, 184106.

13 H. Paulsen and A. X. Trautwein, Top. Curr. Chem., 2004, 235, 197.

14 S. Zein, G. S. Matouzenko and S. A. Borshch, J. Phys. Chem. A, 2005, 109, 8568.

15 G. Lemercier, N. Bréfuel, S. Shova, J. A. Wolny, F. Dahan, M. Verelst, H. Paulsen, A. X. Trautwein and J.-P. Tuchagues, Chem.-Eur. J., 2006, 12, 7421.

16 H. O. Jeschke, L. A. Salguero, B. Rahaman, C. Buchsbaum, V. Pashchenko, M. U. Schmidt, T. Saha-Dasgupta and R. Valentí, New J. Phys., 2007, 9, 448.

17 M. Nishino, K. Boukheddaden, Y. Konishi and S. Miyashita, Phys. Rev. Lett., 2007, 98, 247203; S. Miyashita, Y. Konishi, M. Nishino, H. Tokoro and P. A. Rikvold, Phys. Rev. B: Condens. Matter Mater. Phys., 2008, 77, 014105.

18 L. Kabalan, S. F. Matar, M. Zakhour and J. F. Létard, Z. Naturforsch., B: J. Chem. Sci., 2008, 63, 154.

19 S. Lebègue, S. Pillet and J. G. Ángyán, Phys. Rev. B: Condens. Matter Mater. Phys., 2008, 78, 024433.

20 Y. Chumakov, G. S. Matouzenko, S. A. Borshch and A. Postnikov, Polyhedron, 2009, 28, 1955.

21 C. P. Slichter and H. G. Drickamer, J. Chem. Phys., 1972, 56, 2142.

22 M. Sorai and S. Seki, J. Phys. Chem. Solids, 1974, 35, 555.

23 J.-A. Real, M. C. Muñoz, J. Faus and X. Solans, Inorg. Chem., 1997, 36, 3008.
24 A. J. Pertsin and A. I. Kitaigorodskii, The Atom-Atom Potential Method, Springer-Verlag, Berlin, 1987.

25 MOLCRYST, a package for calculating structure, thermodynamics and phonon spectra of molecular crystals with use of atom-atomic scheme, http://qcc.ru/ netlab.

26 G. Filippini and A. Gavezzotti, Acta Crystallogr., Sect. B: Struct. Sci., 1993, 49, 868.

27 A. K. Rappé, C. J. Casewit, K. S. Colwell, W. A. Goddard III and W. M. Skiff, J. Am. Chem. Soc., 1992, 114, 10024.

28 A. V. Sinitskiy, A. L. Tchougréeff, A. M. Tokmachev and R. Dronskowski, 2009, arXiv:0904.2742v1 [physics.chem-ph].

29 B. Gallois, J.-A. Real, C. Hauw and J. Zarembowitch, Inorg. Chem., 1990, 29, 1152.

30 J.-A. Real, B. Gallois, T. Granier, F. Suez-Panamá and J. Zarembowitch, Inorg. Chem., 1992, 31, 4972.

31 J. D. Dunitz, E. F. Maverick and K. N. Trueblood, Angew. Chem., Int. Ed. Engl., 1988, 27, 880.

32 K. Chandrasekhar and H. B. Bürgi, Acta Crystallogr., Sect. B: Struct. Sci., 1984, 40, 387.

33 P. Gütlich, Y. Garcia and H. Spiering, in Magnetism: Molecules to Materials IV, ed. J. S. Miller and M. Drillon, Wiley-VCH, New York, 2002, ch. 8.

34 H. Lueken, Magnetochemie. Teubner, Stuttgart, 1999.

35 P. Ganguli, Ph. Gütlich, E. W. Müller and W. Irler, J. Chem. Soc., Dalton Trans., 1981, 441.

36 A. V. Sinitskiy, A. L. Tchougréeff and R. Dronskowski. In preparation.

37 M. Kepenekian, B. Le Guennic and V. Robert, Phys. Rev. B: Condens. Matter Mater. Phys., 2009, 79, 094428.

38 A. L. Tchougréeff, A. V. Soudackov, I. A. Misurkin, H. Bolvin and O. Kahn, Chem. Phys., 1995, 193, 19. 